\title{
A twist in the geometry of rotating black holes: seeking the cause of acausality
}

\author{
Hajnal Andréka • István Németi • \\ Christian Wüthrich
}

Received: 16 August 2007 / Accepted: 10 December 2007 / Published online: 9 January 2008

(C) Springer Science+Business Media, LLC 2007

\begin{abstract}
We investigate Kerr-Newman black holes in which a rotating charged ring-shaped singularity induces a region which contains closed timelike curves (CTCs). Contrary to popular belief, it turns out that the time orientation of the CTC is opposite to the direction in which the singularity or the ergosphere rotates. In this sense, CTCs "counter-rotate" against the rotating black hole. We have similar results for all spacetimes sufficiently familiar to us in which rotation induces CTCs. This motivates our conjecture that perhaps this counter-rotation is not an accidental oddity particular to Kerr-Newman spacetimes, but instead there may be a general and intuitively comprehensible reason for this.
\end{abstract}

Keywords Kerr-Newman spacetime - Closed timelike curves ·

Rotating black holes - Time travel

\section{Introduction}

In the present note we investigate rotating black holes and other generally relativistic spacetimes where rotation of matter might induce closed timelike curves (CTCs), thus allowing for a "time traveler" who might take advantage of this spacetime structure.

\footnotetext{
H. Andréka · I. Németi

Rényi Institute of Mathematics, Budapest 1364, Hungary

e-mail: andreka@renyi.hu

I. Németi

e-mail: nemeti@renyi.hu

C. Wüthrich $(\varangle)$

Department of Philosophy, University of California at San Diego,

9500 Gilman Drive, La Jolla, CA 92093-0119, USA

e-mail: wuthrich@ucsd.edu
} 
Most prominently, we will discuss Kerr-Newman black holes in which a rotating charged ring-shaped singularity induces a region which contains CTCs. Due to the electric charge of the singularity, this region is not confined to within the analytic extension "beyond the singular ring", but extends into the side of the ring-singularity facing the asymptotically flat region, from whence the daring time traveler presumably embarks upon her journey. Interestingly, some kind of "counter-rotational phenomenon" occurs here. If a potential time traveler wants to use our Kerr-Newman black hole for traveling into her past, she will have to orbit along the CTC in the direction opposite to that of the rotation of the black hole. In technical words, the time orientation of the CTC is opposite to the direction in which the singularity or the ergosphere rotates.

This state of affairs is at odds with the qualitative, intuitive explanations for the mechanism creating CTCs presented in most popular books. This will motivate the questions formulated in Sect. 4. ${ }^{1}$ We have similar results for all spacetimes sufficiently familiar to us in which rotation induces CTCs. This motivates our conjecture that perhaps this counter-rotation is not an accidental oddity particular to Kerr-Newman spacetimes, but instead there may be a general and intuitively comprehensible reason for this.

Understanding what we take to be the most promising candidate mechanism to produce CTCs in an otherwise causally well-behaved spacetime, i.e. the counterrotational phenomenon mentioned above, is of paramount importance in evaluating the causal stability of generally relativistic spacetimes. Since one possibility to violate Hawking's chronology protection conjecture or, more generally, the strong form of Penrose's cosmic censorship conjecture is through the emergence of acausal features via such a mechanism [8], the present paper contributes to efforts directed at the larger projects of understanding chronology protection and cosmic censorship in general relativity. Furthermore, the issues discussed here are also motivated by discussions in our works [9] and [20]. In those papers it turned out that studying the geometry of rotating black holes can be relevant to some far-reaching considerations in the foundation of mathematics and logic. The counter-rotational phenomenon in KerrNewman spacetime was already noted explicitly in [28, p. 55], albeit without further analysis. A fascinating book providing a broad perspective for the presently discussed matters is Earman [6].

\section{A counter-rotational phenomenon in Kerr spacetime}

We use the standard (Boyer-Lindquist) coordinates $t, r, \varphi, \theta$ for Kerr spacetime which appear e.g. in Hawking-Ellis [16, p. 161], O’Neill [21, pp. 57-59], Wald [27, p. 313], Misner-Thorne-Wheeler [19, p. 877, item (33.2)]. Of these four coordinates, $t, r$ range over the reals, i.e. $-\infty<t, r<\infty$ while $\varphi, \theta$ are spherical coordinates. In pictures, the radius $r$ is drawn as $e^{r}$, so $r=-\infty$ is at the center of the figure and the $r$ coordinate is negative within the sphere indicated in the drawing, see Fig. 1.

\footnotetext{
1 References to popular books offering such intuitive, but misleading, explanations will also be given in Sect. 4.
} 


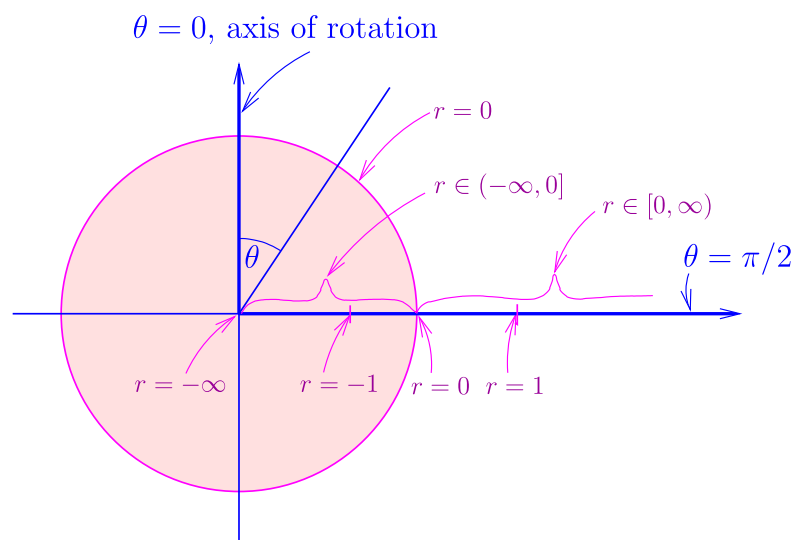

Fig. 1 Illustrating coordinates $r$ and $\theta$. Radius $r$ is drawn as $e^{r}$, so that the shaded circle covers the negative values for $r$

Using these coordinates, the metric tensor field $g$ of the Kerr spacetime is given by

$$
\begin{aligned}
g_{t t} & =-1+2 M r / \rho^{2}, \text { where } \rho^{2}=r^{2}+a^{2} \cos ^{2} \theta, \\
g_{r r} & =\rho^{2} / \Delta, \text { where } \Delta=r^{2}-2 M r+a^{2}, \\
g_{\theta \theta} & =\rho^{2}, \\
g_{\varphi \varphi} & =\left(r^{2}+a^{2}+\left[2 M r a^{2} \sin ^{2} \theta\right] / \rho^{2}\right) \sin ^{2} \theta, \\
g_{t \varphi} & =-2 M r a \sin ^{2} \theta / \rho^{2} \text { and all the other } g_{i j} \text { 's are zero. }
\end{aligned}
$$

Here $a$ denotes the angular momentum per unit mass of the rotating ring, while $M$ is called its mass, cf. e.g. [21, p. 58].

We will concentrate on the so-called "slow-Kerr" case when $0<a^{2}<M^{2}$. In this case there are two event horizons defined by the roots of $\Delta=0$ :

$$
r_{ \pm}=M \pm \sqrt{M^{2}-a^{2}}
$$

In case the Kerr black hole spins sufficiently fast $\left(a^{2}>M^{2}\right)$, these event horizons vanish and we would be faced with a naked singularity. Since such beasts may be regarded as unphysical, we disregard the "fast-Kerr" case. ${ }^{2}$ We will be interested in the "innermost" region of the black hole, defined by $r<r_{-}$, which is where the CTCs (i.e. closed timelike curves) are. This part of the spacetime is called block III or negative exterior Kerr spacetime (EKN-). The so-called "equatorial plane" is defined by $\theta=\pi / 2$. This plane contains, in block III, the so-called ring-singularity

$$
\Sigma=\{\langle t, r, \varphi, \theta\rangle: r=0 \text { and } \theta=\pi / 2\}
$$

\footnotetext{
2 However, everything in this paper applies to the fast-Kerr case, too, except that some formulations would need to be adapted in order to equally apply to the fast-Kerr case.
} 
For the Kerr case, i.e. for an uncharged rotating black hole, the CTCs transpire inside and close to this ring-singularity, i.e. CTCs are found in regions where $r$ is negative but has small absolute value. This part of the spacetime belongs to what [21] calls the Time Machine. ${ }^{3}$ On the other hand, the part outside the ring-singularity and sufficiently close to it belongs to the so-called "ergosphere." The ergosphere (in block III) is defined to be the place where the vectors $\partial_{t}$ parallel to the "time-axis" are not timelike but spacelike. An ergosphere, thus, is a region of spacetime where no observer can remain still with respect to the coordinate system in question. For static black holes, such as those described by Schwarzschild spacetime, the outer limit of the ergosphere coincides with the black hole's event horizon. This is no longer the case when the black holes revolve. Then, the faster the black hole rotates, the more the ergosphere grows beyond the outer horizon $r=r_{+}$. The $(r, \varphi)$-surfaces close to the equatorial plane, i.e. the surfaces with fixed $t$ and fixed small $\cos \theta$, look similar, except that they contain no singularity (if $\cos \theta \neq 0$ ).

All our investigations below are strongly connected to the time-orientation of the spacetime being discussed. Therefore, we recall for convenience that in O'Neill's book [21, p. 76 lines 7-8, p. 60 Definition 2.1.2.] the time-orientation for block III of Kerr spacetime is defined by the vector-field

$$
V(p):=\left(a^{2}+r^{2}\right) \partial_{t}(p)+a \partial_{\varphi}(p) .
$$

With the metric and a time-orientation at hand, the notion of a well-parameterized future-pointing curve makes sense now.

Assume that the lifeline of a particle $\alpha$ is given by the well-parameterized, futurepointing curve $\alpha(\tau)$ with $\tau \in I$ where $I$ is an interval of the reals. We say that $\alpha$ is rotating in the direction $\partial_{\varphi}$ (at $\tau_{0} \in I$ ) if with increasing proper time $\tau$ the values of the $\varphi$-component are increasing (at $\tau_{0}$ ), i.e. if $d \alpha_{\varphi}(\tau) / d \tau$ is positive (at $\tau_{0}$ ). Sloppily, we could write this as " $d \varphi / d \tau>0$ " on $\alpha$ (at $\left.\tau_{0}\right)$. We say that the direction of rotation of $\alpha$ (at $\tau_{0}$ ) is $-\partial_{\varphi}$ if the latter value is negative.

It is known that any particle in the ergosphere must rotate in the $\partial_{\varphi}$ direction, it is not possible to avoid rotating or to rotate in the $-\partial_{\varphi}$ direction in the ergosphere (see e.g. [21, Lemma 2.4.4]). The reason is that in the ergosphere the light cones are tilted in the $\varphi$ direction, i.e. the light cones in the $(t, \varphi)$-cylinders look like those in the left-hand side of Fig. 2.

The situation is drastically different in the "Time Machine" region! There the light cones look like those in the right-hand side of Fig. 2. This means that here it is possible to orbit in the $-\partial_{\varphi}$ direction as well as in the positive $\partial_{\varphi}$ direction, but any time-traveler (i.e. one with $d t / d \tau \leq 0$ ) has to rotate in the $-\partial_{\varphi}$ direction, for it is the only possibility to construct a path with $d t / d \tau<0$ (cf. Fig. 2).

Our above statements can be formulated as saying that the ergosphere and the "Time Machine" rotate in opposite directions. By this we mean that a traveler in

\footnotetext{
3 The notion of a time machine, which has previously been used rather loosely in the physics literature, has recently been subjected to a more rigorous analysis in [7] and [8]. In the full understanding of the vagueness of O'Neill's terminology, we stick to it for simplicity of discussion, as our main issue here is not the question of what should qualify as a time machine.
} 


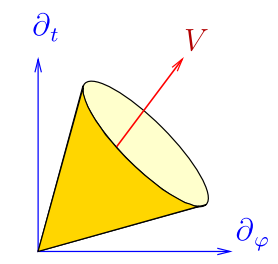

Light cones in the ergosphere

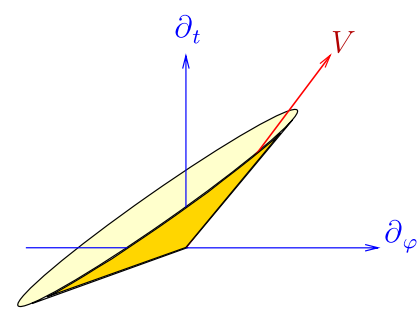

Light cones on the CTCs

Fig. 2 Light cones in the ergosphere and light cones on the CTCs look different

the ergosphere and a traveler moving forward in time in the Time Machine but just preparing for entering a CTC, i.e. with $d t / d \tau>0$ approaching $(d t / d \tau=0)$, move in opposite directions. The one in the ergosphere co-moves with the singularity (or the source) while the one on the CTC, or almost on the CTC, moves in the opposite direction. This results from considering the singularity as the source of the field and the assumption that it rotates in the positive $\varphi$ direction because the total angular momentum $J$ of the Kerr spacetime with $a>0$ is positive. ${ }^{4}$

This is just what we dub a counter-rotating effect: the property that the CTCs counter-rotate with the ring-singularity (and with the ergosphere) in the sense just described. In other words, the time-orientations of the CTCs point in the $-\partial_{\varphi}$ direction while the ergosphere rotates in the opposite, $+\partial_{\varphi}$ direction. We note that this counterrotational effect remains valid if we extend our attention beyond the equatorial plane defined by $\theta=\pi / 2$. We also note that this counter-rotational effect does not depend on which of the two possible time-orientations we choose.

We can formulate this counter-rotational effect in a coordinate-independent way by saying that where the (invariantly defined) Killing vector field $\partial_{\varphi}$ is timelike, its time-orientation is negative (and hence a would-be time traveler must orbit in the $-\partial_{\varphi}$ direction). Here we assume that the time-orientation is chosen such that the rotation of the source points in the positive $\varphi$ direction. (We note that our claim that the CTCs and the ergosphere rotate in the opposite directions can also be formulated in a coordinateindependent way.)

\section{Counter-rotation in Kerr-Newman spacetime}

At this point one might be tempted to think that perhaps the cause of the above counterrotational phenomenon might be found in the fact that the co-rotating area (ergosphere) and the counter-rotating area (Time Machine) are separated by the ring-singularity. So perhaps the counter-rotation can be explained by saying that the ring-singularity acts like a mirror turning directions into their negatives. Such a symmetry about the ring-singularity would still not account for the rotational sense of the ring-singularity itself; in particular it would keep us wondering why the singularity co-rotates with the ergosphere but counter-rotates with the CTCs. The simplest way of seeing that such mirroring about the singular region cannot possibly give a hint for the diametric

$\overline{4 \text { For details, cf. [21, pp. 58,179] }}$ [27, pp. 296-297, 314]. 
revolutions of the ergosphere and the Time Machine is by looking at Kerr-Newman spacetimes where the co-rotational and counter-rotational areas are no longer separated by the ring-singularity. ${ }^{5}$

Kerr-Newman spacetimes describe black holes with angular momentum as in the Kerr case, but with an electric charge in addition. We are using the definition of KerrNewman spacetime as given e.g. in Misner-Thorne-Wheeler [19, pp. 877-881, e.g. item (33.2) on p. 877], Wald [27, p. 313, item (12.3.1)], d'Inverno [5, p. 264, item (19.72)], or Wüthrich [28].

The Kerr-Newman metric can be obtained from the Kerr metric (in Boyer-Lindquist coordinates) by simply replacing all occurrences of $2 \mathrm{Mr}$ with $\left(2 \mathrm{Mr}-e^{2}\right)$, where $e$ is the electric charge of the black hole. ${ }^{6}$ Thus, the metric will take on the form

$$
\begin{aligned}
g_{t t} & =-1+\left(2 M r-e^{2}\right) / \rho^{2}, \quad \text { where } \rho^{2}=r^{2}+a^{2} \cos ^{2} \theta, \\
g_{r r} & =\rho^{2} / \Delta, \quad \text { where } \Delta=r^{2}-2 M r+e^{2}+a^{2}, \\
g_{\theta \theta} & =\rho^{2}, \\
g_{\varphi \varphi} & =\left(r^{2}+a^{2}+\left[\left(2 M r-e^{2}\right) a^{2} \sin ^{2} \theta\right] / \rho^{2}\right) \sin ^{2} \theta, \\
g_{t \varphi} & =\left(e^{2}-2 M r\right) a \sin ^{2} \theta / \rho^{2} \text { and all the other } g_{i j} \text { 's are zero. }
\end{aligned}
$$

The vector field $V(p)$ as defined in Sect. 2 is an admissible time-orientation for block III of the Kerr-Newman spacetime, too. As the focus of the present article is precisely on block III, this reassurance suffices for our purposes. The counter-rotating phenomenon described in the previous section holds in the case of charged KerrNewman holes, too. However, this counter-rotation effect is more poignant than in the Kerr case, for the following reason. In the Kerr-Newman spacetime there are CTCs both at small positive values of $r$ (say, at $r=0.1 \mathrm{M}$ ) and also at small negative values of $r$ (say, at $r=-0.1 \mathrm{M}$ ) in the equatorial plane. Thus, the ergosphere and the "Time Machine" are no longer separated by the ring-singularity in this case. However, here, too, the time-orientation of the CTC at $r=0.1 \mathrm{M}$ points in the direction $-\partial_{\varphi}$, i.e. in the negative $\varphi$ direction. Using our terminology introduced above, this means that a "time-traveler" inhabiting the CTC at $r=0.1 \mathrm{M}$ orbits in the direction opposite to the rotation of the massive ring. In other words, the CTC and the ring-singularity "rotate" in opposite directions just as they did in the Kerr case, as shown in Fig. 3.

Moreover, all CTCs in Kerr and Kerr-Newman spacetimes counter-rotate with the massive ring. In particular, any ring-shaped CTC like $\mathcal{R}(\mathbf{r})=\{\langle 0, r, \varphi, \theta\rangle: \theta=$ $\pi / 2$ and $r=\mathbf{r}$ ) for fixed $\mathbf{r}$ has time-orientation $-\partial_{\varphi}$ (for all possible choices of $\mathbf{r}$ making $\mathcal{R}(\mathbf{r})$ into a CTC). ${ }^{7}$

\footnotetext{
5 For completeness we note that a further possibility for seeing this is staying with Kerr spacetime and focusing attention to $(r, \varphi)$ hypersurfaces with fixed, small values of $\theta$. In such a hypersurface the co-rotating and counter-rotating regions still exist but they are no longer separated by a singularity.

6 This statement is a refinement of what [21] writes on p. 61 about the connection between the two metrics.

7 We note that Wüthrich [28, Chap. 6, pp. 75-85] is highly relevant to our investigations here (e.g. it is proved there that in order to remain on a CTC, in Kerr-Newman spacetime, the "time-traveler" has to use a prohibitive amount of fuel). Furthermore, Figures 4.2 and 4.4 on pages 49 and 52 in [28] depict the causality violating regions for the Kerr and the Kerr-Newman case, respectively.
} 


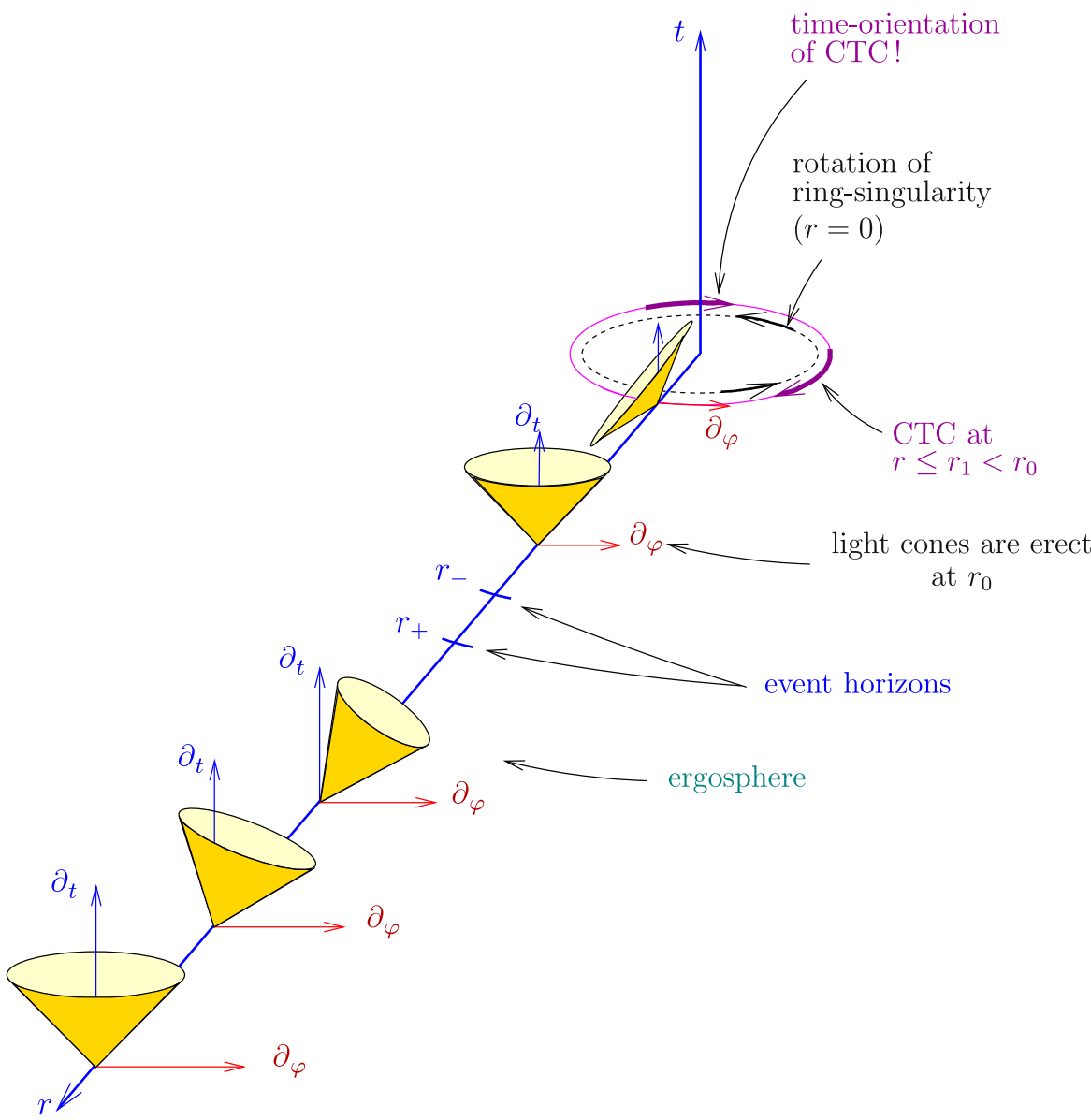

Fig. 3 This is how light cones behave in Kerr-Newman spacetimes. For $r_{0}, r_{1}$ and computations see Sect. 5, especially Fig. 4

\section{Seeking explanations}

In the literature, the apparently standard account for why and how rotating matter induces CTCs goes as follows. ${ }^{8}$ Given, for example, a Kerr-Newman black hole, let us mentally move toward the singularity starting from a point far away from the black hole. Let us further assume that this vantage point is situated on the equatorial plane of the black hole. The Kerr-Newman spacetime is asymptotically flat. Thus, at this distant

\footnotetext{
8 Cf. e.g. Gribbin [14, pp. 145-152, and Fig. 8.1 on p. 151] or Gribbin [15, pp. 214-220 (e.g. Figs. 7.5, 7.6 on p. 215,218 resp.)]. Gribbin [15] writes on the bottom of page 217 that "[t]he critical stage for the light cone tipping, as far as time travel is concerned, is when the cone is tipped by more than 45 degrees. Since the half-angle of the cone is $45^{\circ}, \ldots$.'. Further references for the standard explanation are e.g. Paul Davies [4, first 3 pages of Sect. 2], Nick Herbert [17, Fig. 6-2 on p. 105], Paul Horwich [18, p. 113, Fig. 28], and Clifford A. Pickover [22, Fig. 14.2 (p. 185), Fig. 17.1 on p. 224 might also be relevant].
} 
point, the black hole does not influence the light cones significantly and we can safely assume that they are straight or "vertical" like in Minkowski spacetime. Let us then move slowly towards the black hole. As it gets closer, the so-called dragging of inertial frames caused by the rotation of the black hole comes into action. This dragging effect is explained in relativity textbooks via Mach's principle, e.g. [27, pp. 319, 187, 89] or [19, pp. 547, 879, 1117-1120]. So as an influence of the rotation of our massive ring (the singularity), the light cones get tilted in the direction $\varphi$ of the rotation of the ring. Naturally, the closer we get to the rotating ring, the stronger this dragging effect, and thus the tilting, will be. The standard account goes on by saying that eventually the light cones tip over completely (i.e. they dip below the equatorial plane), so they become approximately "horizontal," making $\partial_{\varphi}$ timelike (and $\partial_{t}$ spacelike). This clearly leads to CTCs by $\partial_{\varphi}$ being timelike. This literature thus creates the false impression that the proper time of the CTCs co-rotates, rather than counter-rotates, with the matter content of the universe. The time orientation of the CTCs, according to this "official story," therefore agrees with the direction of rotation of the source of the field.

It is important to stress that according to this official story the time orientation of the so obtained CTCs agrees with the direction $\varphi$ of rotation of the black hole-contrary to what has been established in Sects. 2 and 3. Hence a time traveler using such CTCs would orbit in the positive $\varphi$ direction, i.e. would rotate in the same direction as the black hole does. If this explanation worked, it would yield an intuitively convincing, natural explanation for why and how the basic principles of general relativity lead in certain situations (such as when appropriately distributed rotating masses are present) to CTCs. One of our main points is that the above explanation does not work, simply because light cones behave differently in the relevant spacetimes, as is also illustrated in Fig. 3.

Let us see how the corrected story goes based on detailed computations in KerrNewman spacetime. These computations will be presented in Sect. 5. First of all, we emphasize that the new, corrected story suggested in the present paper does not offer any kind of explanation for the creation of CTCs. Instead, it merely offers a description of the behavior of the light cones. We maintain, however, that a proper understanding of this behavior, and particularly the counter-rotation that we are interested in, at least constitutes an important and promising first step toward such an explanation. For simplicity we assume that the charge and rotation of the black hole are sufficient for ensuring that there are CTCs at positive values of the radius $r$, i.e. outside the ringsingularity. Surely enough, the corrected story begins exactly like the official party line: distant light cones are erect, i.e. vertical and they start to tilt in the $\varphi$ direction as we begin moving towards the black hole. But this tilting effect does not grow beyond any limit as we move towards the ring-singularity. As we move inward (towards the ring), the tilting grows for a while but then it stops growing, and eventually at a radius $r_{0}$ safely outside the time-travel region, the light cone (in the $t \varphi$-plane) is erect again. From $r_{0}$ inward, the tilting is in the other direction, i.e. in the $-\varphi$ direction (Until now, i.e. at values greater than $r_{0}$, the tilting was always in the $+\varphi$ direction). Thus, tilting in the $\varphi$ direction did not result in CTCs, because $r_{0}$ is safely outside the time-travel region. Moreover, tilting alone in the $-\varphi$ direction does not lead to CTCs, either, because from $r_{0}$ inward, the time-axis is always inside the light cone (i.e. $\partial_{t}$ is always timelike). 
So what happens if we go closer and closer to the ring-singularity? In other words, what creates the CTCs if not the cone tipping? The answer is that, eventually, the light cones open up in the negative $\varphi$ direction. This second effect is carried to such an extreme that $-\partial_{\varphi}$ becomes contained in the future light cone, for sufficiently small but positive values of the radius $r$. If we are close enough to the ring-singularity, then time travel becomes possible-but only if we orbit in the negative $\varphi$ direction. Hence the time traveler has to orbit in the direction opposite to the rotation of the black hole. It is this fact that we refer to as the phenomenon of counter-rotating. Let us summarize the new story's two most important features. These are:

(i) counter-rotation (i.e. the time orientation of the CTCs is $-\partial_{\varphi}$ ), and

(ii) it is not the tilting of the light cones due to the dragging of inertial frames which leads to the formation of CTCs, but a second effect, primarily resulting in their opening up in the negative $\varphi$ direction.

The open issue we would like to raise is the following. The official story as presented at the beginning of this section provided an intuitive physical account as to what causes the tipping of the light cones. To repeat, our computations show that this official story is not true and that it has to be replaced with the new story outlined above. Unfortunately, however, we cannot offer an equally intuitive and suggestive explanation for the new story. Hence, we would like to issue a challenge to our readers in form of the following question:

Question 1 Is there a qualitative - and similarly compelling — explanation as to why the time traveler has to "counter-rotate" against the rotating ring-singularity in KerrNewman spacetime? Can one find a physical mechanism which qualitatively explains why and how CTCs are "created" by rotation of matter?

In the above description of the "new story" we concentrated on Kerr-Newman black holes for simplicity. The situation is similar in many spacetimes where rotation of relatively large masses leads to the formation of CTCs. For example, our corrected story applies equally to Kerr black holes, with appropriate modifications to adapt the train of thought to the fact that in the Kerr case the CTCs only transpire in the negative radius region. ${ }^{9,10}$

\footnotetext{
9 To see the counter-rotation effect in action in the Kerr case, our metaphorical spaceship must approach the causality-violating region somewhat "from above" the equatorial plane, for it would crash into the ring-singularity otherwise. In other words, we choose some fixed $\theta$ with $\cos \theta>0$ which, however, must be sufficiently small for intersecting the causality violating region. This slight change of itinerary does not alter in principle the counter-rotational effect we discussed at length for the Kerr-Newman case.

10 Despite the seemingly widely held presupposition that the CTCs co-rotate rather than counter-rotate with the black hole, we seem to have an ally in Brandon Carter, at least as far as the Kerr case is concerned. He seems to concur with our conclusion that the outlined counter-rotation does not arise from a coordinate artefact, but constitutes a tangible physical process when he writes that "in order to make up literally for the lost time, the path must enter the region [where CTCs transpire]. Here time can be gained but only at the expense of clocking up a large change (negative for $a>0$ ) in the angle $\hat{\varphi}$." [3, p. 1566, below item (28); our emphasis]. A second ally is Robert L. Forward [10] who on p. 172 describes time travel via Kerr spacetime as "travelling near the rotating ring in the direction against rotation of the ring for a number of rotations". Cf. also his Fig. 10 on p. 176.
} 


\section{Computations}

In this section, we present computations supporting the claims made in Sect. 4. We do the computations for the Kerr-Newman metric; we assume $a \neq 0, e \neq 0$ and $M>\sqrt{a^{2}+e^{2}}$. We are in the equatorial plane, i.e. $\theta=\pi / 2, \cos \theta=0$, and $\sin \theta=1$. We are interested in what the light cones look like in the $t \varphi$-planes, as a function of the radius $r$, and we are interested in positive $r$ only as this suffices to study the tilting and widening of the light cones. Using the shorthand

$$
\begin{aligned}
& E=\left(2 M r-e^{2}\right) / r^{2} \quad \text { we have } \\
& g_{t t}=E-1, \quad g_{t \varphi}=-a E, \text { and } g_{\varphi \varphi}=r^{2}+a^{2}+a^{2} E .
\end{aligned}
$$

These functions are depicted in Fig. 4.

Let us first check where the CTCs are. We have CTCs where $g_{\varphi \varphi}$ is negative, and this is exactly where

$$
G(r):=r^{2} g_{\varphi \varphi}=r^{4}+a^{2} r^{2}+2 a^{2} M r-a^{2} e^{2}
$$

is negative. The radial derivative of $G$ is $G^{\prime}=4 r^{3}+2 a^{2} r+2 a^{2} M$, this is everywhere positive because $r \geq 0$ and $M>0$. Hence $G$ is monotonically increasing (as we move from 0 to infinity). We have $G(0)=-a^{2} e^{2}<0$ and $G$ tends to infinity as $r$ tends to positive infinity; hence there is a unique value $r_{1}$ where $G$ is zero, above which $G$ is positive and below which $G$ is negative. Hence the same is true for $g_{\varphi \varphi}$. Thus $r_{1}$ (which is the root of $g_{\varphi \varphi}$ ) is the place where CTCs "appear", and we will be interested in seeing what the light cones do as we move towards $r_{1}$ from greater values of $r$.

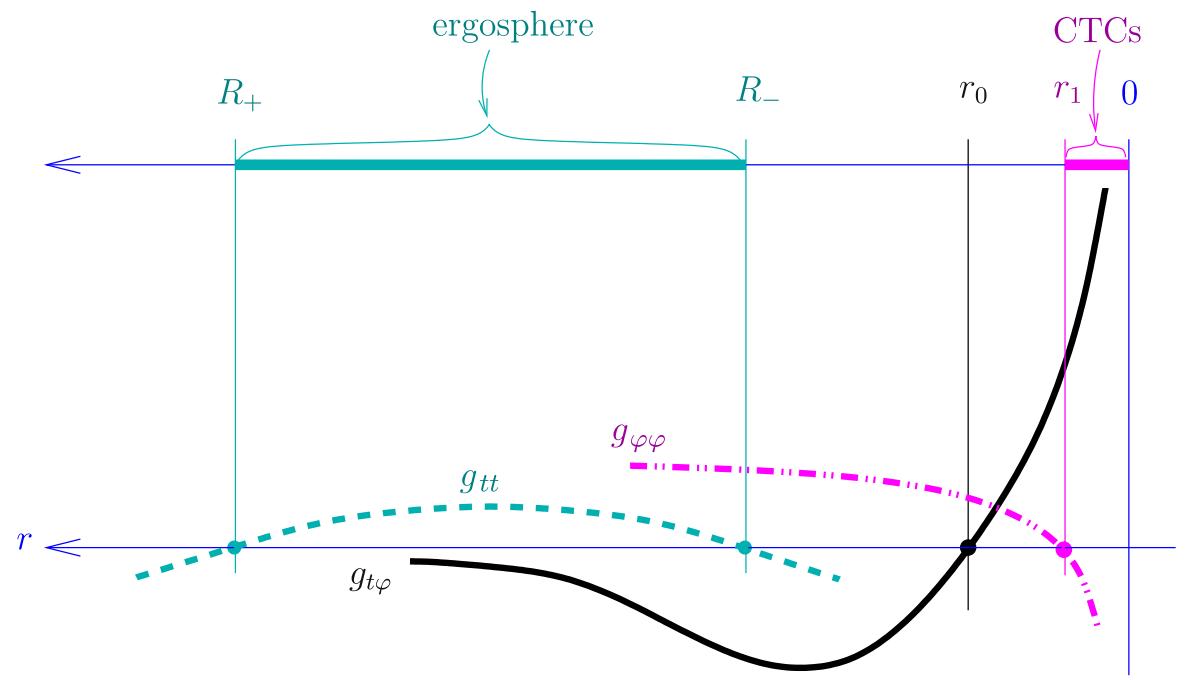

Fig. 4 Illustration for the Kerr-Newman metric. The picture faithfully represents the order of the roots (i.e. zeros) of $g_{t \varphi}, g_{t t}$ and $g_{\varphi \varphi}$ and where these three functions are negative or positive; the picture does not faithfully represent the proportions of the distances and their magnitudes. The ergosphere and the "Time Machine" (where the CTCs are) are disjoint, and the light cone is erect between them at $r_{0}$. In some sense, $g_{t \varphi}$ represents the tilting of the light cones. See also Fig. 3 
In order to see how the light cones tilt and widen in the $t \varphi$-planes, we want to know for what values $y$ is the direction $v=\partial_{t}+y \partial_{\varphi}$ lightlike, i.e. for which $y$ we have $g(v, v)=0$. These values are the solutions of the equation

$$
\begin{aligned}
& y^{2} g_{\varphi \varphi}+2 y g_{t \varphi}+g_{t t}=0, \text { hence } \\
& y=\left(-g_{t \varphi} \pm \sqrt{g_{t \varphi}^{2}-g_{\varphi \varphi} g_{t t}}\right) / g_{\varphi \varphi} . \text { Let } \\
& c:=\left(-g_{t \varphi}\right) / g_{\varphi \varphi}, \text { for center of light cone, } \\
& d:=\left(\sqrt{g_{t \varphi}^{2}-g_{\varphi \varphi} g_{t t}}\right) / g_{\varphi \varphi}, \text { for half-diameter of light cone, see Fig. 5. }
\end{aligned}
$$

The light cone is erect, i.e. is not tilted, where $c=0$. This is where $E=0$, i.e. at $r_{0}:=e^{2} / 2 M$. At $r=r_{0}$ we have $E=0$, thus $g_{t t}=-1, g_{t \varphi}=0$, and $g_{\varphi \varphi}=r_{0}^{2}+a^{2}$. This is almost the same as in Minkowski spacetime, the only difference being that $g_{\varphi \varphi}$ is not $r^{2}$, but a bit larger. Hence the light cones at $r_{0}$ are "standing", but they are a bit narrower than at infinity (the bigger $a$ is, the narrower the light cones). See also Fig. 3.

We show that $r_{0}>r_{1}$. Since the function $G(r)=r^{2} g_{\varphi \varphi}$ decreases monotonically as we approach 0 and $G\left(r_{1}\right)=0$, in order to show $r_{0}>r_{1}$ it is enough to show $G\left(r_{0}\right)>0$, and in order to show this it is enough to show $g_{\varphi \varphi}\left(r_{0}\right)>0$ (since $\left.r_{0} \neq 0\right)$. That this is the case can easily be seen:

$$
g_{\varphi \varphi}\left(r_{0}\right)=r_{0}^{2}+a^{2}+a^{2} E=r_{0}^{2}+a^{2}=\left(e^{4} / 4 M^{2}\right)+a^{2}>0 \text { since } a \neq 0 .
$$

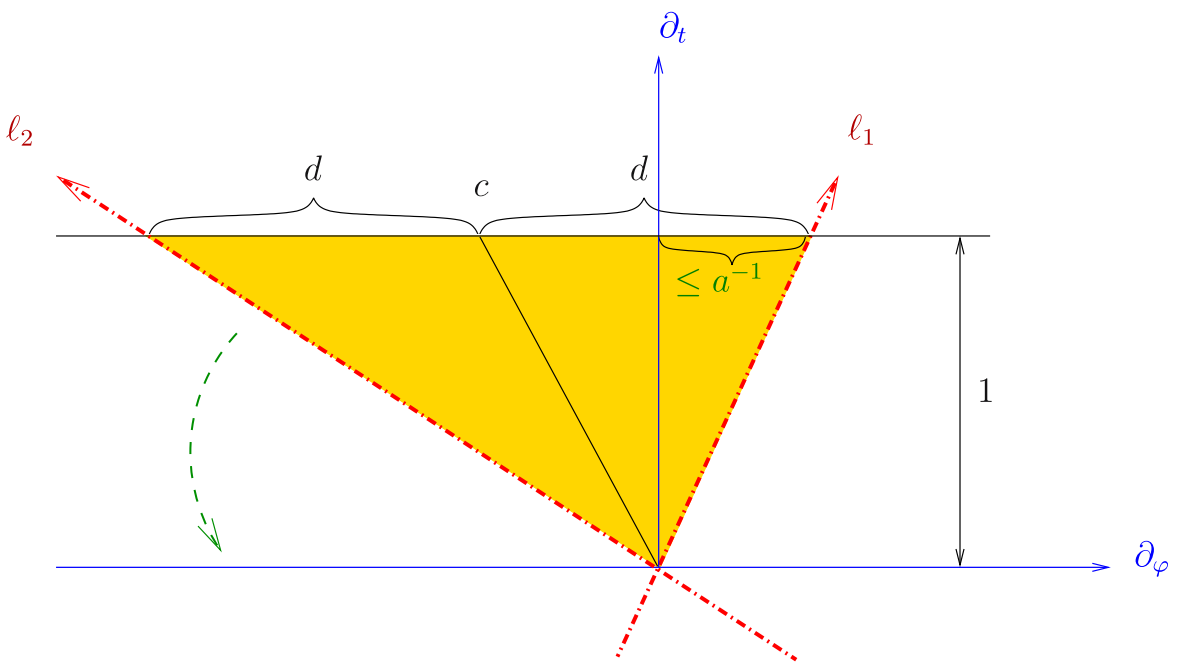

Fig. 5 The light cone in the $t \varphi$-plane, between $r_{0}$ and $r_{1}$. We draw the light cones with respect to the Killing vector fields $\partial_{t}, \partial_{\varphi}$. As we move towards the time machine region, i.e. towards $r_{1}, \ell_{2}$ lowers to horizontal while $\ell_{1}$ is less than or equal $a^{-1}$ 
Let us have a look at the roots of $g_{t t}$. The smaller root of $g_{t t}$, i.e. $R_{-}=M-$ $\sqrt{M^{2}-e^{2}}$, is bigger than $r_{0}$, because of the following:

$$
\begin{aligned}
& \frac{e^{2}}{2 M}<M-\sqrt{M^{2}-e^{2}} \quad \text { iff } \\
& \frac{e^{2}}{2 M}\left(M+\sqrt{M^{2}-e^{2}}\right)<\left(M-\sqrt{M^{2}-e^{2}}\right)\left(M+\sqrt{M^{2}-e^{2}}\right) \quad \text { iff } \\
& \frac{e^{2}}{2 M}\left(M+\sqrt{M^{2}-e^{2}}\right)<e^{2} \quad \text { iff } \\
& M+\sqrt{M^{2}-e^{2}}<2 M, \quad \text { which always holds for } e \neq 0 .
\end{aligned}
$$

The ergosphere is between $R_{+}$and $R_{-}$. With $R_{-}>r_{0}>r_{1}$ we thus have shown that the light cones are erect somewhere between the ergosphere and the "Time Machine" (as we claimed in Sect. 4). We note that the two event horizons are at $r_{ \pm}=M \pm$ $\sqrt{M^{2}-e^{2}-a^{2}}$ (these are the roots of $\Delta=r^{2}-2 M r+e^{2}+a^{2}$ ). For $r_{+}, r_{-}$and $r_{0}, r_{1}$ see also Fig. 3. We have established the following, see Fig. 4:

$$
R_{+}>r_{+}>M>r_{-}>R_{-}>r_{0}>r_{1}>0
$$

We are interested in the behavior of the light cones as we move from $r_{0}$ towards $r_{1}$. We have just shown that in this interval the time-axis is always timelike, i.e. that it is within the light cone. We will now establish the following statements concerning the light cones as depicted in Fig. 5 as we move from $r_{0}$ to $r_{1}$ :

(1) the center $c$ of the light cone moves from zero to minus infinity, monotonically,

(2) the diameter of the light cone grows to infinity, monotonically,

(3) the right side of the light cone is always "slower" than $a^{-1}$.

As a consequence of (1) and (2), the left side of the light cone lowers towards the horizontal plain. We could summarize these statements as "the light cone tilts in the negative $\varphi$ direction and opens up".

To prove (1) and (2), first we show that

$$
g_{\varphi \varphi}=r^{2}+a^{2}+a^{2}\left(2 M r-e^{2}\right) / r^{2}
$$

decreases monotonically as we move from $r_{0}$ towards $r_{1}$. Its radial derivative is

$$
\left(g_{\varphi \varphi}\right)^{\prime}=2\left[r+a^{2}\left(e^{2}-M r\right) / r^{3}\right] .
$$

By $0<r \leq r_{0}=\frac{e^{2}}{2 M}<\frac{e^{2}}{M}$ we have $\left(g_{\varphi \varphi}\right)^{\prime} \geq 0$. Thus, $g_{\varphi \varphi}$ decreases monotonically as we move from $r_{0}$ to $r_{1}$. Furthermore, the reader is reminded that $g_{\varphi \varphi}$ is positive in the open interval $] r_{1}, r_{0}\left[\right.$ and zero for $r_{1}$.

Now, as can be seen in Fig. $4,-g_{t \varphi}$ is negative and decreases monotonically as we move from $r_{0}$ to $r_{1}$. Hence $c=-g_{t \varphi} / g_{\varphi \varphi}$ is negative and approaches monotonically minus infinity as we move from $r_{0}$ to $r_{1}$. This proves (1).

To prove (2), we first compute:

$$
\begin{aligned}
g_{t \varphi}^{2}-g_{\varphi \varphi} g_{t t} & =a^{2} E^{2}-\left(r^{2}+a^{2}+a^{2} E\right)(E-1)=r^{2}+a^{2}-E r^{2} \\
& =r^{2}+a^{2}+e^{2}-2 M r .
\end{aligned}
$$


This is always positive for $r \leq r_{0}$, and since it is a second-order polynomial in $r$ whose roots are larger than $r_{0}$, it increases as we move from $r_{0}$ to $r_{1}$. The same is true for the square root of the above expression. Since $g_{\varphi \varphi}$ decreases monotonically, this shows that the "diameter" of the light cone increases monotonically as we move from $r_{0}$ towards $r_{1}$. It approaches infinity because $r_{1}$ is the root of $g_{\varphi \varphi}$. Thus, (2) has been proved.

It remains to show that $c+d \leq a^{-1}$, i.e. $y_{1} \leq a^{-1}$ where $y_{1}:=\left(-g_{t \varphi}+\right.$ $\left.\sqrt{g_{t \varphi}^{2}-g_{\varphi \varphi} g_{t t}}\right) / g_{\varphi \varphi}$. Let us use the notation $S:=\sqrt{g_{t \varphi}^{2}-g_{\varphi \varphi} g_{t t}}$. Now

$$
\begin{aligned}
y_{1} & =\left(-g_{t \varphi}+S\right) / g_{\varphi \varphi}=\left(S-g_{t \varphi}\right)\left(S+g_{t \varphi}\right) /\left(g_{\varphi \varphi}\left(S+g_{t \varphi}\right)\right) \\
& =\left[\left(g_{t \varphi}^{2}-g_{\varphi \varphi} g_{t t}\right)-g_{t \varphi}^{2}\right] /\left[g_{\varphi \varphi}\left(S+g_{t \varphi}\right)\right] \\
& =-g_{t t} /\left(S+g_{t \varphi}\right) .
\end{aligned}
$$

We are interested in the behavior of the above expression as we move $r$ from $r_{0}$ towards $r_{1}$. When substituting the values for $g_{t t}$ and $g_{\varphi \varphi}$ we get

$$
\begin{aligned}
y_{1} & =\left(r^{2}-2 M r+e^{2}\right) /\left[r^{2} S+a e^{2}-2 M a r\right] \\
& \leq\left(r^{2}-2 M r+e^{2}\right) /\left(a r^{2}-2 M a r+a e^{2}\right)=a^{-1}
\end{aligned}
$$

because $S=\sqrt{r^{2}+a^{2}+e^{2}-2 M r} \geq a$ if $r \leq r_{0}$. With this, (3) has been proved.

\section{A generic phenomenon?}

We have seen in Sects. 4 and 5 that if you want to time travel in a Kerr-Newman spacetime, you have to orbit around the $\theta=0$ axis in the direction opposite to the rotation carried out by the singularity. In other words, a time traveler must counterrotate with the singularity. The same happens in Kerr spacetime, see Sect. 3. As it turns out, however, the phenomenon of counter-rotation is not limited to these important classes of spacetimes.

The same counter-rotation phenomenon is present in van Stockum's rotating dust solution [26], in the Tipler-van Stockum fast-rotating cylinder [24,25], and in Gödel's rotating universe [13]. Counter-rotation in the first two examples is discussed in [2], for counter-rotation in the third example see [1]. Moreover, the same phenomenon is also present in the case of Gott's cosmic strings based CTCs, cf. [11], [12, Fig. 14, p. 108]. There the CTCs counter-rotate with the system formed by the pair of strings.

In sum, thus, in at least five of the most prominent examples of spacetimes involving CTCs, the future direction on the CTCs opposes the rotational sense of the source of the gravitational field. ${ }^{11}$

\footnotetext{
11 In case of asymptotically flat spacetimes (like Kerr-Newman ones) the counter-rotating effect can be formulated in an invariant way as we did in Sect. 2 in this paper. For the rest of our examples, we use slightly different invariant formulations. For example, in Gödel's spacetime an invariant formulation can be obtained by following Gödel's wording in [13, p.271, lines 1-10]. Gödel uses gyroscopes as "compasses of inertia" in the sense of [19] and Rindler [23, p. 197, under the name "gyrocompass"].
} 
This raises a question: Is this counter-rotation an accident or is it a mathematical or at least physical necessity in some sense, e.g. under some suitable physical assumptions, all of which must of course be satisfied by the five examples above? One of these assumptions is that the CTCs in question are "created" by rotation of matter, i.e. rotation of the gravitational source. In its present form this question is somewhat loose and rather programmatic. Let us thus give a more tangible formulation of this question.

Question 2 In general, how important is it for the CTCs to counter-rotate against the rotational sense of the gravitating matter which brings about the CTCs? In particular, is there any example of a spacetime where the CTCs are generated by rotating matter and there is no counter-rotation effect?

We believe that our calculations and our arguments above and in [2] not only admit the relevance and interest in asking this question, but moreover, strongly suggest that there might well be a general principle at work: a principle which states that CTCs generated by rotating matter must spiral in the opposite direction from the rotational sense of the matter. We are not sure how much work such a principle would offer toward a general understanding of the mechanisms which bring about spacetime structures that contain CTCs. But it strikes us as an observation which is potentially crucial for such an endeavor, particularly because the phenomenon seems so pervasive in such an important set of spacetimes with CTCs.

Acknowledgments We would like to express our thanks to John Earman for helpful discussions and encouragement. We would like to express our gratitude for very helpful discussions at several points in the past to Attila Andai, Gábor Etesi, Judit X. Madarász, Endre Szabó and László B. Szabó. Very special thanks go to Renáta Tordai for various help, e.g. careful reading. We would like to thank two anonymous referees for helpful suggestions. C.W. thanks the Department of Philosophy at the University of Bern for its hospitality. This research was supported by the Hungarian National Foundation for scientific research grant No. T43242.

\section{References}

1. Andréka, H., Madarász, J.X., Németi, I.: Visualizing some ideas about Gödel-type rotating universes. Rényi Institute Budapest. Manuscript (2002)

2. Andréka, H., Németi, I., Wüthrich, C.: CTCs and counter-rotation in the spacetime of the Tipler-van Stockum infinite rotating cylinder (in preparation)

3. Carter, B.: Global structure of the Kerr family of gravitational fields. Phys. Rev. 174(5), 1559-1571 (1968)

4. Davies, P.: How to Build a Time Machine. Viking Penguin, New York (2002)

5. d'Inverno, R.: Introducing Einstein's Relativity. Clarendon Press, Oxford (1992)

6. Earman, J.: Bangs, Crunches, Whimpers, and Shrieks. Singularities and acausalities in relativistic spacetimes. Oxford University Press, Oxford (1995)

7. Earman, J., Smeenk, C., Wüthrich, C.: Take a ride on a time machine. Available online in the PhilSci archive, http://philsci-archive.pitt.edu/archive/00001673/

8. Earman, J., Wüthrich, C.: Time Machines. In: Zalta, E. (ed.) The Stanford Encyclopedia of Philosophy, Winter 2004 edition

9. Etesi, G., Németi, I.: Non-Turing computability via Malament-Hogarth space-times. Int. J. Theor. Phys. 41(2), 341-370 (2002)

10. Forward, R.L.: Future Magic. Avon Books, New York (1988)

11. Gott, J.R.: Closed timelike curves produced by pairs of moving cosmic strings: exact solutions. Phys. Rev. Lett. 66, 1126-1129 (1991) 
12. Gott, J.R.: Time Travel in Einstein's Universe. Houghton Mifflin Co., Boston (2001)

13. Gödel, K.: Lecture on rotating universes. In: Feferman, S., Dawson, J.W., Goldfarb, W., Parsons, C., Solovay, R.N. (eds.) Kurt Gödel Collected Works, vol. III, pp. 261-289. Oxford University Press, New York (1995)

14. Gribbin, J.: Spacewarps. Penguin Books (1983)

15. Gribbin, J.: In search of the edge of time (Black holes, white holes, wormholes.) Penguin Books, 1992 (1998)

16. Hawking, S.W., Ellis, G.F.R.: The large scale structure of space-time. Cambridge Monographs on Mathematical Physics, Cambridge University Press, Cambridge (1973)

17. Herbert, N.: Faster Than Light. New American Library books (1988)

18. Horwich, P.: Asymmetries in Time. The MIT Press, Cambridge (1988)

19. Misner, C.W., Thorne, K.S., Wheeler, J.A.: Gravitation. W. H. Freeman an Company, New York (1970)

20. Németi, I., Dávid, Gy.: Relativistic computers and the Turing barrier. J. Appl. Math. Comput. 178, 118-142 (2006)

21. O'Neill, B.: The Geometry of Kerr Black Holes. A K Peters Wellesley, Massachusetts (1995)

22. Pickover, C.A.: Time. Oxford University Press, Oxford (1998)

23. Rindler, W.: Relativity, Special, General and Cosmological. Oxford University Press, Oxford (2001)

24. Tipler, F.J.: Rotating cylinders and the possibility of global causality violation. Phys. Rev. D 9(8), 22032206 (1974)

25. Tipler, F.J.: Causally symmetric spacetimes. J. Math. Phys. 18(8), 1568-1573 (1977)

26. van Stockum, W.J.: The gravitational field of a distribution of particles rotating about an axis of symmetry. Proc. R. Edinb. 57, 135-154 (1937)

27. Wald, R.M.: General Relativity. The University of Chicago Press, Chicago (1984)

28. Wüthrich, C.: On time machines in Kerr-Newman spacetime. Master Thesis, University of Berne, (1999). http://www.itp.unibe.ch/diploma_thesis/wuthrich/wuthrichLiz.pdf 